Fontan procedure, includes connection of the vena cava (VC) to the pulmonary artery (PA) using a bio-inert conduit to reduce work required by the right ventricle $(\mathrm{RV})$. While this operation greatly extends the lives of HLHS patients, the Fontan circuit eventually fails, and the only solution is a scarcely available donor heart. This failed circuit is explained by the "Fontan paradox" where central venous pressures build up over time, causing increased systemic resistance and congestion. The absence of the sub-pulmonary ventricle leads to abnormal hemodynamics associated with lifethreatening complications. We believe that decreasing central venous pressures through the use of a tissue engineered contractile, patient specific conduit will decrease the amount and severity of complications caused by the "Fontan paradox." We will use amniotic fluid derived induced pluripotent stem cells (AF-iPSCs) differentiated into cardiomyocytes (CMs) to generate flow within a biodegradable conduit. Additionally, AF-iPSC will be differentiated into structural support cells (SSCs), including cardiac fibroblasts and epicardium. Several studies suggest advanced contraction and structure of CMs in specific ratios with SSCs, particularly mouse and human fetal fibroblasts. In combination, these cells have shown advanced tissue organization and function through mechanically and electrically aligned junctions. This allows them to have a magnitude higher contractile force than CMs alone, making them ideal for increasing pressure within a tissue engineered construct. This poster focuses on the differentiation and selection of SSCs. METHODS/STUDY POPULATION: AF-iPSCs differentiation began at roughly $80 \%$ confluency. Mesoderm formation occurred via WNT pathway modulation by supplementing RPMI+insulin media with $0.5 \mathrm{ng} / \mathrm{mL} \mathrm{BMP} 4$ at day 0 , followed by $3 \mathrm{ng} / \mathrm{mL} \mathrm{BMP} 4$, $2 \mathrm{ng} / \mathrm{mL}$ Activin A, and $5 \mathrm{ng} / \mathrm{mL}$ BFGF for four days. Then, RPMI+insulin media was supplemented with $10 \mathrm{ng} / \mathrm{mL}$ of BMP4 until day fifteen for epicardial formation. Cells were lifted to induce epithelial-to-mesenchymal transition (EMT) and RPMI-insulin media was supplemented with $10 \mathrm{ng} / \mathrm{mL}$ BFGF for cardiac fibroblasts. They were then harvested and characterized using immunofluorescence. Planned experiments include RT-qPCR for further characterization of cardiac fibroblasts. Additionally, a fibroblast isolation plating technique will be utilized to obtain cardiac fibroblast from AF-iPSC CMs and AF-iPSC epicardium. Commercially obtained human cardiac fibroblasts will be utilized as a control for all studies. RESULTS/ANTICIPATED RESULTS: Immunofluorescence (IF) revealed positive expression of vimentin and $\alpha$-SMA indicating a fibroblast and vascular smooth muscle phenotype after supplementation with $10 \mathrm{ng} / \mathrm{mL}$ of BMP4 after EMT induction. It is expected that IF of epicardial formation at day 15 will show positive expression of WT1, a well-known epicardial marker. We also suspect RT-qPCR will reveal high expression of cardiac fibroblast specific markers COL1A1, PDGFA, TCF21, and THSB1. We expect to yield a higher number of cardiac fibroblast from the small molecule AF-iPSC differentiation compared to a timed plating technique of AF-iPSC CMs and AF-iPSC epicardium (separately plated). Results will be quantified and compared using the aforementioned techniques. DISCUSSION/ SIGNIFICANCE OF IMPACT: Discussion/significance of impact: Although fibroblasts make up a large portion of cells in the heart and greatly enhance CM function, they are poorly characterized in the literature and not easily obtained. This study will provide an efficiency comparison on the best method for acquiring cardiac fibroblast for cardiac tissue engineering applications as we move forward with translational cardiac pediatric research.
Development of human engineered cardiac tissue (hECT)-based screening assay to explore cardiac contractile properties in response to pharmacological challenge with proarrhythmic drugs

Francesca Stillitano, Irene C. Turnbull, Jaydev Dave, Jean-Sébastien Hulot and Roger J. Hajjar

Mount Sinai School of Medicine; Icahn School of Medicine at Mount Sinai; Institute of Cardiometabolism and Nutrition (ICAN); Icahn School of Medicine at Mount Sinai

OBJECTIVES/SPECIFIC AIMS: The goals of this study were (1) to evaluate the effect of proarrhythmic drugs on calcium transient and (2) to use three-dimensional human engineered cardiac tissue (hECT) technology to evaluate cardiac contractile properties in response to pharmacological challenge with proarrhythmic drugs. METHODS/ STUDY POPULATION: Calcium transient was measured in subjectspecific iPSC-CMs by using the IonOptix system in Sotalol treated vs. untreated conditions. We fabricated human engineered cardiac tissues (hECT) in a custom designed bioreactor using low- and high-sentitive subject-specific iPSC-CMs. Contractile function of the hECT was evaluated at baseline and after Sotalol $[300 \mu \mathrm{M}]$ administration. The change in beat rate was recorded under spontaneous beating conditions; changes in other twitch parameters, including time to relaxation, were recorded under electrical stimulation. Time to relaxation served as an indicator of action potential duration (APD), which has a temporal correlation with the QT interval. RESULTS/ ANTICIPATED RESULTS: The low-sensitive iPSC-CM showed a considerable drop in overall peak height of the calcium transient, in the presence of $100 \mu \mathrm{M}$ Sotalol. The high-sensitive line, however, showed a more pronounced drop in peak height. Sotalol treatment also induced a more pronounced increase in the exponential decay time constant (tau) in the high-sensitive line compared to the lowsensitive line. The hECT fabricated with high sensitive hiPSC-CM showed a larger decrease in spontaneous beat rate in response to Sotalol ( 0.41 vs 0.23 fold decrease), with a higher increase in time to relaxation (1.8 vs 1.3 fold increase), compared to hECT from low sensitive hiPSC-CM. Moreover, while the low-sensitive hECT showed a positive correlation between time to relaxation and developed force, as expected after Sotalol stimulation; the high-sensitive hECT failed to show a positive inotropic response. DISCUSSION/ SIGNIFICANCE OF IMPACT: Our findings suggest subject-specific iPSC-CMs and hECT, can be used to model functional abnormalities observed in diLQTS in response to Sotalol, and offer novel insights into human-based screening assays for toxic drug reactions. Success of this study may help identify key components underlying diLQT susceptibility to ultimately develop novel therapeutic agents.

3521

Distinct single cell gene expression in peripheral blood monocytes correlates with treatment response groups to TNF-alpha inhibition in rheumatoid arthritis

Theresa Wampler Muskardin ${ }^{1}$, Zhongbo $\mathrm{Jin}^{2}$, Jessica M. Dorschner ${ }^{3}$, Yogita Ghodke-Puranik ${ }^{1}$ and Timothy Niewold ${ }^{1}$

${ }^{1}$ New York University - $\mathrm{H}+\mathrm{H}$ Clinical and Translational Science Institute; ${ }^{2}$ University of Florida and ${ }^{3}$ Mayo Clinic

OBJECTIVES/SPECIFIC AIMS: The cellular mechanisms that underlie the IFN $\beta / \alpha$ ratio that predicts response are not known. Effects of IFN on single immune cells may be masked in whole blood 
or mixed cell populations. By studying the effect of IFN $\beta / \alpha$ activity ratio on individual monocytes, we can determine the functional impact of the IFN ratio and suggest the cellular mechanisms that underlie response/non-response to TNFi therapy in RA. METHODS/STUDY POPULATION: We used single cell analysis to investigate whether monocyte gene expression differs significantly between RA patients according to their pre-TNFi serum IFN- $\beta / \alpha$ ratio. Single classical (CL) and non-classical (NC) blood-derived monocytes were isolated from 15 seropositive RA subjects prior to biologic therapy. Subjects were grouped by pre-TNFi serum IFN- $\beta / \alpha$ ratio into two groups, those with a high IFN- $\beta / \alpha$ ratio $(\geq 1.3, n=6)$ and those with a low IFN- $\beta / \alpha$ ratio $(<1.3, n=9) .87$ target genes were analyzed. Genes that varied significantly between the groups by categorical analyses were tested in multivariate logistic regression models. RESULTS/ANTICIPATED RESULTS: Every participant was seropositive for rheumatoid factor and antibodies to cyclic citrullinated peptide. Among the participants in the groups, there were no significant differences in age or DAS scores $(\mathrm{P}>0.05)$. The treatments were comparable and none were being treated with biologic therapy. There were striking differences in monocyte gene expression between patients with pre-treatment blood IFN $\beta$ / $\alpha$ activity $<1.3$ and $\geq 1.3$. Expression of (1) key type I IFN pathway genes (JAK1, STAT2, IFIT2, IFIH1, PRDM1); (2) IL12; (3) CD36; and (4) CTLA4 were the strongest differentiators between groups ( $\mathrm{p}<0.0001$ for each, corrected for multiple comparisons). DISCUSSION/SIGNIFICANCE OF IMPACT: In this study we were able to measure gene expression in single monocytes from seropositive RA patients prior to biologic treatment. Within-cell co-expression patterns demonstrate biological differences in monocytes of RA patients with an IFN $\beta / \alpha \geq 1.3$, the ratio of type I IFNs which predicts non-response to TNFi. The data suggest that there may be differential IFN production and pathway activation in patients who do not respond to TNFi. The increased expression of CD36 in monocytes from RA patients with high IFN $\beta / \alpha$ activity may be a reflection of increased "foam cells" in the inflamed tissue of patients who do not respond to TNFi. Enrichment of CTLA4 in those with high serum IFN $\beta / \alpha$ suggests that CTLA4-Ig may be less likely to be an effective alternative for someone who is not likely to respond to TNFi. Current work includes determining whether the peripheral blood findings reflect altered cellular composition, type I IFN production and signaling in the synovium. Significance: This work will help to develop a more individualized approach to therapy in RA and determine an immunological basis of response/ non-response to TNFi.

\section{Dynamic Afterload Cardiac Microtissue Model To Examine Molecular Pathways of Heart Failure}

Abhinay Ramachandran ${ }^{1}$, Carissa Livingston ${ }^{1}$, Elise Corbin ${ }^{1}$, Alexia Vite ${ }^{1}$, Alex Bennett ${ }^{1}$ and Kenneth Margulies ${ }^{1}$

${ }^{1}$ University of Pennsylvania School of Medicine

OBJECTIVES/SPECIFIC AIMS: This project aims to determine the key molecular pathways that link increased myocardial wall stress to cardiomyocyte hypertrophy and subsequent heart failure. We will use a cardiac microtissue (CMT) model with dynamically tunable cantilever stiffness to examine changes in CMT hypertrophy and electro-mechanical properties in response to increased afterload (cantilever stiffness). Subsequently, we will determine if inhibition of pro-hypertrophic or anti-hypertrophic pathways alter the hypertrophic response to increased afterload. Primary outcomes for this study are static/dynamic force, minimum electric field strength (VT), maximum capture rate (MCR), average cell area, and tissue crosssectional thickness, and secondary outcomes are degree of myoblast activation and apoptosis. METHODS/STUDY POPULATION: CMT platforms will be fabricated using iron-doped polydimethylsiloxane (PDMS) to create magnetically tunable cantilevers. Cantilever stiffness will be increased with the application of an external magnetic field. Cantilever stiffness will be measured using a capacitance probe, where the force required to deflect both the cantilever and calibration probe is in accordance with Hooke's Law. Human induced pluripotent stem cell cardiomyocyte (hiPSC-CMs) will be cultured and matured as 3D CMTs. In-vitro static/dynamic force generation will also be calculated by measuring the deflection of the cantilevers and applying Hooke's law. CMTs will be paced using carbon electrodes to obtain VT and MCR. Structural data will be obtained using immunostaining and confocal microscopy. Finally, we will use pharmacologic inhibitors to inhibit molecular pathways that we identified in prior genetic screens such as ABCC8 (anti-hypertrophic mediator) and C1QTNF9 (pro-hypertrophic mediator). We will examine each of these pathways in low- and high-stiffness conditions. RESULTS/ ANTICIPATED RESULTS: We believe increased afterload will cause significant hypertrophy, measured by increases in CMT crosssectional thickness, cardiac myocyte area, myofibroblast activation, and myocyte apoptosis. In addition, we expect to see increases in static/dynamic force, increased voltage threshold, and decreased maximum capture rate. Preliminary results show a $64.3 \%$ increase in force generation when stiffness is increased by approximately $30 \%$, and a $44.4 \%$ decrease in force generation when stiffness is decreased by approximately $30 \%$. Finally, we expect that inhibiting a pro- or anti-hypertrophic molecular pathway will weaken or strengthen the hypertrophic response to increased afterload, respectively. DISCUSSION/SIGNIFICANCE OF IMPACT: To our knowledge, our lab is the first to create a dynamically tunable afterload system in the cantilever CMT model. This advance provides us with a robust platform to determine the molecular pathways that cause increased myocardial wall stress to result in cardiomyocyte hypertrophy and heart failure, which remain a critical knowledge gap in our understanding of cardiovascular disease. With more precise understanding of these pathways, we will equip ourselves with the knowledge to develop novel therapeutic agents to prevent the development or progression of heart failure.

3004

Effects of Early Life Stress on Adult Behavioral and Neural Outcomes in Rats

Alexandra Moussa-Tooks ${ }^{1}$, Ken Mackie², John Green ${ }^{3}$, Lisa Bartolomeo ${ }^{3}$, Alex Gimeno ${ }^{3}$, Eric Larson ${ }^{3}$, Heather Bradshaw ${ }^{3}$, Emma Leishman ${ }^{3}$, Brian O'Donnell ${ }^{3}$ and William Hetrick ${ }^{3}$

${ }^{1}$ Indiana University School of Medicine; ${ }^{2}$ Department of Psychology, University of Vermont and ${ }^{3}$ Psychological and Brain Sciences, Indiana University

OBJECTIVES/SPECIFIC AIMS: Early life stress is known to greatly impact neurodevelopment during critical periods, conferring risk for various psychopathologies, including the onset and exacerbation of schizophrenia and anxiety disorders. The endocannabinoid system is highly integrated into the stress response and may be one means by which early life stress produces such deleterious effects. Using a naturalistic, ecologically valid animal model, this study explored interactions between the stress response and endocannabinoid systems within the cerebellum, a region dense with the $\mathrm{CB} 1$ endocannabinoid 